\title{
Compact Ultra-wideband Grounded Coplanar Waveguide to Substrate Integrated Waveguide Tapered V-Slot Transition
}

\author{
Diego Lorente ${ }^{1}$, Markus Limbach ${ }^{1}$, Héctor Esteban ${ }^{2}$ and Vicente Boria ${ }^{2}$
}

\begin{abstract}
In this work, a new ultra-wideband Grounded Coplanar Waveguide to Substrate Integrated Waveguide transition is presented. The proposed design improves the performance of the triangular tapered slot transition, by providing a return loss level better than $28 \mathrm{~dB}$ in the $\mathrm{X}$-Band frequency range (with enhancements of up to $29 \mathrm{~dB}$ at higher frequencies in such a band), and a flatter response for the insertion losses. The analysis of the new transition is performed using a dielectric material with high permittivity $\left(\varepsilon_{r}=9.8\right)$ which leads to an extremely compact implementation. In order to validate the proposed design, several back-to-back transitions with different lengths were manufactured, measured and compared with the simulated data. Further experimental results, after performing an additional Thru-Reflect-Line calibration for de-embedding undesired effects of involved connectors, are also shown.
\end{abstract}

Index Terms-CPW, SIW, electrical interface, transition, compact, performance improvement, TRL calibration

\section{INTRODUCTION}

$\mathbf{I}$ $\mathrm{N}$ the past years, there has been an increasing interest on some demanding applications, such as radar systems, that involves the design of feeding networks for antenna phased arrays, where not only a good electrical performance but also a high density of integration is required, especially for airborne and spaceborne applications [1]. This demands a careful selection of the technology of implementation in order to fulfill both requirements.

For such applications, antennas, power dividers and other RF front-end components are typically designed in planar technologies such as microstrip, thanks to its conformability, low cost and ease of integration. However, the performance of microstrip circuits is strongly dependent on the dielectric substrate losses and its thickness. As an alternative, Coplanar Waveguide (CPW) presents less attenuation as the fields do not entirely propagate within the substrate, being less sensitive to its dielectric losses [2]. In addition CPW also has lower radiation losses. Typical applications based on CPW includes a bottom ground plane. This variation, called Grounded Coplanar Waveguide (GCPW), allows a better integration among other circuits and components.

The Substrate Integrated Waveguide (SIW) has become also a promising technology for numerous applications due to its

1 Microwaves and Radar Institute, SAR-Technology. German Aerospace Center. Münchener Str. 20, 82234 Weßling, Germany, Tel.: +49 8153282703 , E-mail: diego.lorentecatalan@dlr.de

${ }^{2}$ Instituto de Telecomunicaciones y Aplicaciones Multimedia. Universitat Politècnica de València. Camino de Vera, s/n, 46022 Valencia, Spain, Tel.: +34963877007 (Ext. 88278), E-mail: hesteban@dcom.upv.es high quality factor, good shielding and power handling [3]. It presents the advantages of the classic waveguides, but at the same time it allows a high level of compactness typical of planar circuits, by using substrates with high dielectric permittivities.

Therefore, the combination of these two technologies, GCPW and SIW, is a common solution for some demanding applications, whose performance strongly depends on the interface between them [4]. Thus, the design of a transition between GCPW and SIW plays a key role in the electrical behavior of such structures. Some previous transition designs have been made using, respectively, a current probe [5] and a $90^{\circ}$ bent slot [6] based on $\mathrm{H}$-field and E-field coupling, but both of them provide a limited bandwidth. Some other proposals enhance its bandwidth by using a U-shaped slot antenna [7], combining the $90^{\circ}$ bent slot with a steppedimpedance resonator [8] or modifying the stub angle [9]. An ultra-wideband performance can be achieved by means of truncating the GCPW bottom conductor plane and its viaholed areas in different geometries [10], by adding an elevated CPW section [11] or by using a coupling slot with a triangular tapered aperture that serves as an improved impedance transformer [12]. This last solution is widely applied in a broad range of applications ([13]-[15]).

In this work, a new design based on a geometrical variation of the triangular tapered slot is presented. The new V-Slot transition provides a better matching level, showing enhancements of up to $29 \mathrm{~dB}$ at higher frequencies in XBand. The new transition is designed using a high permittivity $\left(\varepsilon_{r}=9.8\right)$ substrate, which leads to an extremely miniaturized implementation of the proposed GCPW-SIW transition.

This work is structured as follows: first, the new V-Slot transition is presented and compared with the triangular tapered slot of [12]. Then, the measurements of two proposed back-to-back V-Slot transitions will be compared with the simulated data. In order to extend the analysis, an additional TRL calibration will be applied to remove the effect of the connectors and adapters from the measurements. Once again the measurements of both manufactured samples will be compared with the simulated data. Finally, the validation of the new transition is discussed in the conclusions.

\section{TRANSITION DESIGN}

The proposed GCPW to SIW transition is a geometrical variation of the triangular tapered coupling slot. The new 
design modifies the slot aperture, including a vertex at the edge, resembling a V-shaped slot. Both transitions with its geometrical parameters are shown in Figure 1. The design values of the GCPW line, transitions and the SIW structure are displayed in Table I.
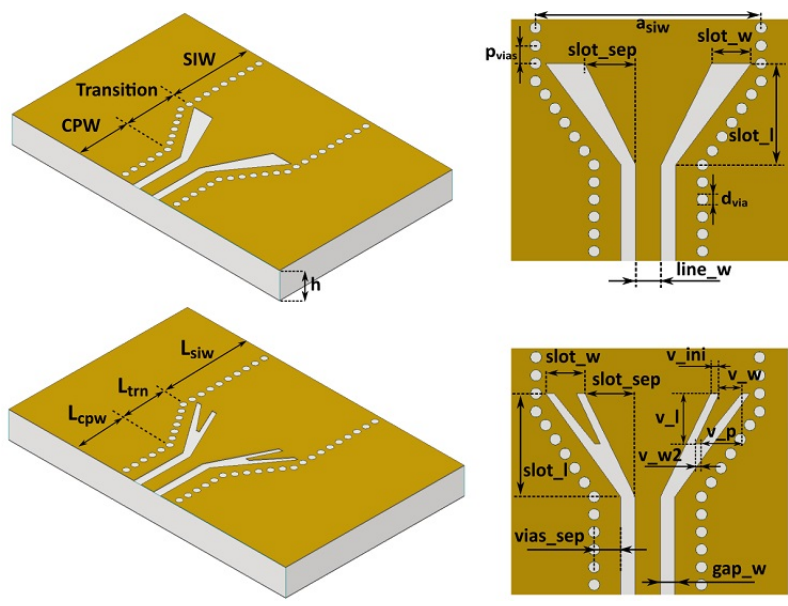

Fig. 1. Design values of the triangular tapered slot (top) and V-Slot (bottom).

TABLE I

DESIGN PARAMETERS (IN MM)

\begin{tabular}{|c|c|c|c|c|c|c|c|}
\hline \multicolumn{2}{|c|}{ CPW values } & \multicolumn{4}{|c|}{ Transition values } & \multicolumn{2}{|c|}{ SIW values } \\
\hline line_w & 0.9 & slot_l & 3.7 & $v \_w$ & 0.8 & $a_{\text {siw }}$ & 8 \\
\hline$g a p \_w$ & 0.52 & slot_w & 1.4 & $v_{-} l$ & 1.8 & $d_{\text {via }}$ & 0.4 \\
\hline vias_sep & 0.95 & slot_sep & 1.8 & $v \_p$ & 1.45 & $p_{\text {vias }}$ & 0.65 \\
\hline$L_{c p w}$ & 3.5 & $v_{-} i n i$ & 0.3 & $v_{-} w 2$ & 0.2 & $L_{\text {siw }}$ & 6.5 \\
\hline
\end{tabular}

Typically the triangular tapered coupling slot presents an optimized length slot_l$\approx \lambda / 4$ in order to maximize its response bandwidth. In the proposed V-Slot, the inner slot length does not remain constant due to the variation of the aperture, thus making it more suitable for smaller wavelengths and providing a better performance for higher frequencies. This new tapering avoids the abrupt slot ending, thus enabling a smoother GCPW to SIW fields conversion.

The feeding GCPW input line is optimized to have a $50 \Omega$ impedance. The value vias_sep is chosen according to [6], in order to avoid the appearance of higher CPW modes. The length of the transition section $L_{t r n}$ is $3.05 \mathrm{~mm}$.

An analysis of the two transitions is performed in X-Band and optimized for a center frequency of $9.6 \mathrm{GHz}$. The designs are implemented using a substrate Rogers TMM10i with a high dielectric permittivity $\left(\varepsilon_{r}=9.8, \tan \delta=0.002\right)$ and thickness $h$ of $1.905 \mathrm{~mm}$. The simulated structure is fed by means of a GCPW input line and finished in SIW, as shown in Figure 1.

The performance of each design is compared in Figure 2. The new V-Slot transition improves the matching level from $4 \mathrm{~dB}$ in the low-frequency range up to $29 \mathrm{~dB}$ at higher frequencies. The new transition also offers less leakage or radiation losses. Regarding its power handling, it also provides lower electric field peak values, which makes it less susceptible to suffer from corona effect (for airborne applications), and multipactor (for satellite application under high vacuum conditions).
The simulation and optimization process of both designs were performed with HFSS 2019 R1.

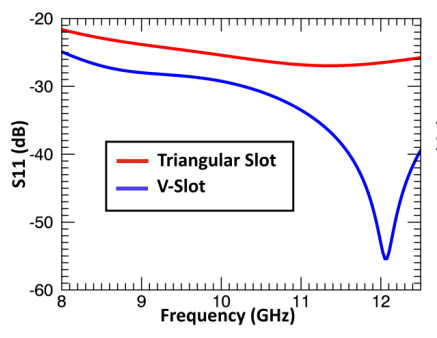

(a) Return loss

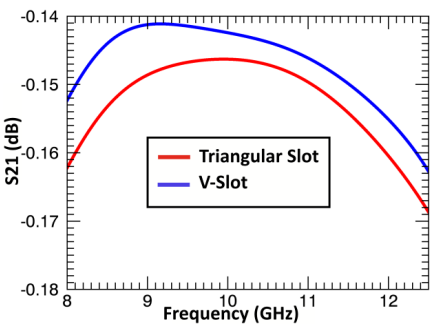

(b) Insertion loss
Fig. 2. Comparison performance GCPW-SIW transitions.

\section{MANUFACTURED BACK-TO-BACK TRANSITIONS}

In order to validate the proposed structure, two back-toback V-Slot transitions with different lengths L1 and L2 were manufactured and measured.

Each GPCW line is fed by means of a $50 \Omega$ SMP connector, a novel type of miniaturized surface mounted SMA [16]. The manufactured back-to-back transitions are shown in Figure 3. The lengths L1 and L2 are $13.1 \mathrm{~mm}$ and $26.2 \mathrm{~mm}$ corresponding, to one and two wavelengths. The values line_cpw, ini_line and $d_{-} f$ are $7 \mathrm{~mm}, 0.87 \mathrm{~mm}$ and $3.8 \mathrm{~mm}$ respectively.

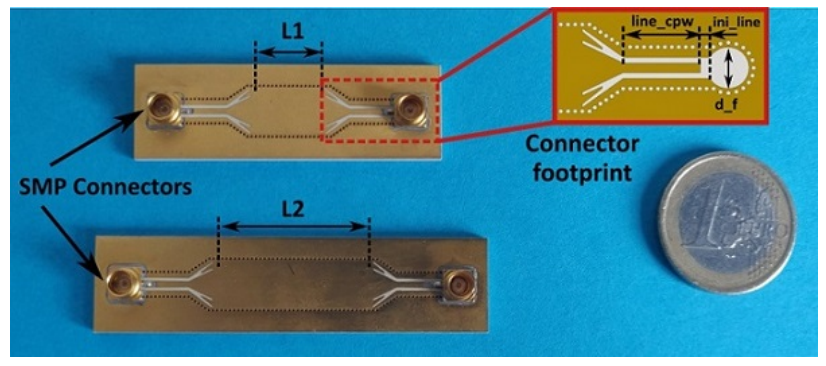

Fig. 3. Manufactured back-to-back V-Slot transitions.

\section{A. Measurement of the manufactured prototypes}

The measurements of the two prototypes and a comparison with the simulated data are shown in Figures 4 and 5. The experimental results show a really good agreement with the simulation. The measured matching level is better than $15 \mathrm{~dB}$ for the entire X-Band, and higher than $20 \mathrm{~dB}$ for the design centre frequency. The transmission factor is $0.3-0.4 \mathrm{~dB}$ lower, due to the insertion losses of the SMA-SMP adapters.

The measured return loss level presents a standing wave due to a slight mismatch, which is produced by the use of the connectors. The appearance of such effect is difficult to avoid, as the measured return loss level is low, and the sensitivity of the structure (due to connector soldering, fabrication tolerances, etc) is more visible in this logarithmic range. The level of the standing wave varies according to the connector's position along the structure, depending on the standing wave location where they are placed. By performing a Thru-ReflectLine Calibration [17], a de-embeding procedure can be done to analyze the transition performance independently on the type of the employed connectors. 


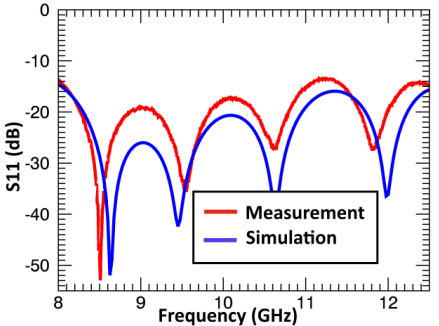

(a) Return loss

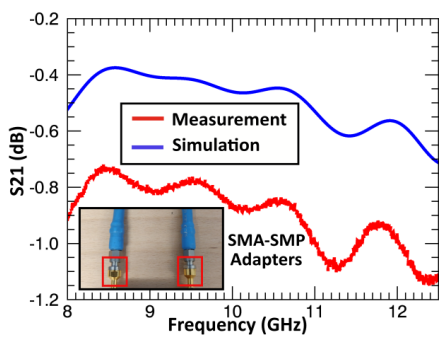

(b) Insertion loss
Fig. 4. Measured back-to-back V-Slot transition with length L1.

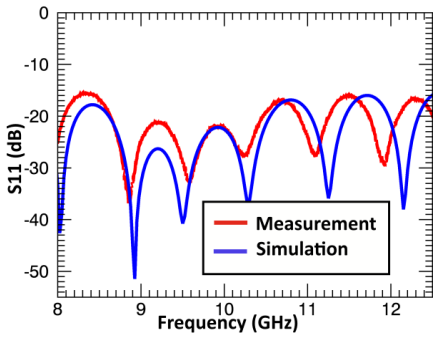

(a) Return loss

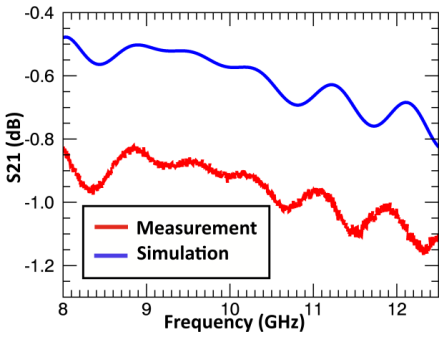

(b) Insertion loss
Fig. 5. Measured back-to-back V-Slot transition with length L2.

\section{B. Analysis of the measurements after applying a TRL Cali- bration}

In order to analyze the measurements regardless of the feeding connectors, a Thru-Reflect-Line (TRL) Calibration is used. This way, the effect of the connectors and the SMA-SMP adapters will be considered in the calibration, thus removing the slight mismatch from the measured data. This procedure requires a calibration kit, composed of a Thru, an Open or Short and a Line. The calibration plane is placed $3.5 \mathrm{~mm}$ before the transition.

In addition, two back-to-back triangular tapered slots transitions of lengths L1 and L2 are also manufactured. All the manufactured prototypes and the calibration kit can be seen in Figure 6. A performance analysis of the triangular tapered slot and V-Slot transition, after applying a TRL Calibration, is shown in Figures 7 and 8.

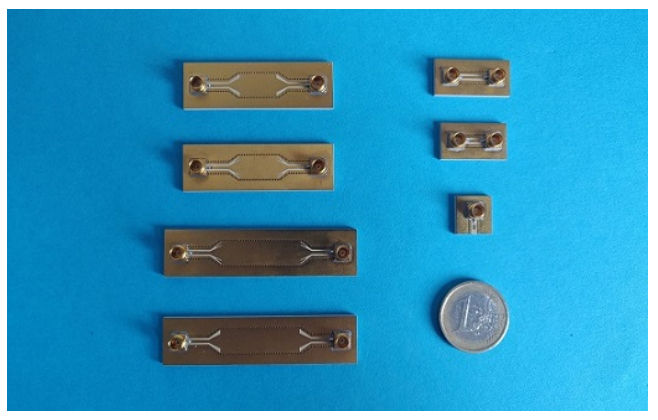

Fig. 6. Manufactured back-to-back transitions (left) and Calibration Kit (right).

It can be seen that the standing wave is no longer present in the measurements. For both prototype lengths, the new V-Slot shows a better overall matching level of up to $7 \mathrm{~dB}$ than the triangular tapered slot, and a flatter insertion loss response.

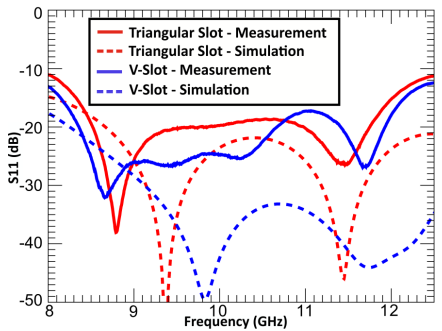

(a) Return loss

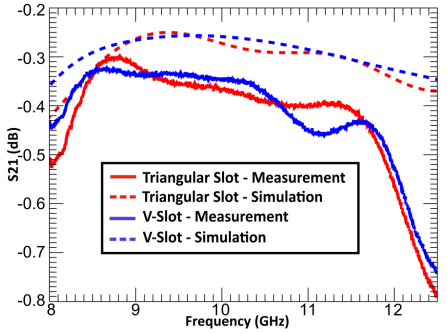

(b) Insertion loss
Fig. 7. Measured back-to-back transitions with length L1 applying TRL Calibration.

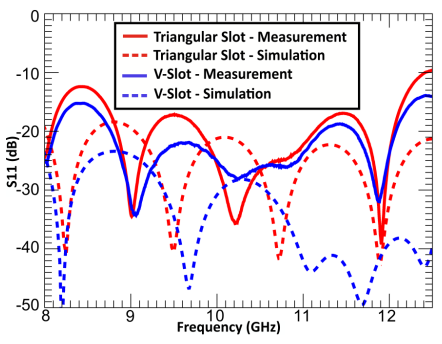

(a) Return loss

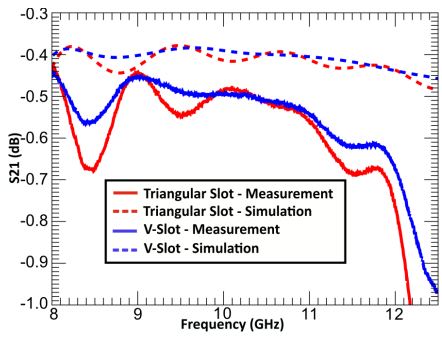

(b) Insertion loss
Fig. 8. Measured back-to-back transitions with length L2 applying TRL Calibration.

However, the simulation results also show an improvement for higher frequencies that is not accomplished in the measurements. Despite its good matching level, it differs from the simulation. These inaccuracies can be explained because of the inhomogeneity in the feeding, especially the connector soldering, which ideally has to be the same for all the manufactured lines and calibration kit, a task difficult to achieve in practice. This effect is also more visible for higher frequencies, since the sensitivity of the calibration procedure increases. Furthermore, the use of a high dielectric substrate makes the structure really sensitive to fabrication tolerances, thus also leading to further discrepancies. As the range values for $\left|S_{11}\right|$ is, to a large extend, below $-20 \mathrm{~dB}$ any small inaccuracy provides a variation of some dBs.

Nevertheless, the above-mentioned disagreement is present for both type of transitions, as the same calibration conditions are applied. Therefore, the enhanced performance of the proposed V-Slot leads to a more robust solution, providing a wider margin to deal with such effects.

\section{CONCLUSION}

In this paper, a compact ultra-wideband V-Slot GCPW-SIW transition is presented. The proposed transition improves the performance of the triangular tapered slot, providing a better overall matching level. The measurements of the manufactured back-to-back V-Slot transitions agree very well with the simulated data. The analysis of the measurements, after applying a TRL calibration, shows also that the new design provides an improved return loss and a flatter insertion loss response. In this case, the disagreement between simulated and measured data is attributed to the strict calibration conditions, which at the same time proves that the new transition is more robust against tolerances and undesired measurement effects. 


\section{REFERENCES}

[1] A. Reigber et al., "DBFSAR: An airborne very high-resolution digital beamforming SAR system," Proceedings of 14th European Radar Conference, Nuremberg, Germany, Oct. 2017.

[2] R. K. Saini, S. Dwari, and M. K. Mandal, "CPW-fed dual-band dualsense circularly polarized monopole antenna," IEEE Antennas and Wireless Propagation Letters, vol. 16, pp. 2497-2500, 2017.

[3] D. Deslandes and K. Wu, "Integrated microstrip and rectangular waveguide in planar form," IEEE Microwave and Wireless Components Letters, vol. 11, no. 2, pp. 68-70, 2011.

[4] R. Kazemi and A. Fathy, "Design of a wide band eight-way compact SIW power combiner fed by a low loss GCPW-to-SIW transition," Progress In Electromagnetics Research C, vol. 26, pp. 97-110, 2012.

[5] D. Deslandes and K. Wu, "Analysis and design of current probe transition from grounded coplanar to substrate integrated rectangular waveguides," IEEE Transactions on Microwave Theory and Techniques, vol. 53, no. 8, pp. 2487-2499, 2005.

[6] A. Patrovsky, M. Daigle, and Ke Wu, "Millimeter-wave wideband transition from CPW to substrate integrated waveguide on electrically thick high-permittivity substrates," in 2007 European Microwave Conference, 2007, pp. 138-141.

[7] G. Gentile, B. Rejaei, V. Jovanović, L. K. Nanver, L. C. N. de Vreede, and M. Spirito, "Ultra-wide band CPW to substrate integrated waveguide (SIW) transition based on a U-shaped slot antenna," in 2013 European Microwave Integrated Circuit Conference, 2013, pp. 25-28.

[8] R. Fang, C. Liu, and C. Wang, "Compact and broadband CB-CPWto-SIW transition using stepped-impedance resonator with $90^{\circ}$-bent slot," IEEE Transactions on Components, Packaging and Manufacturing Technology, vol. 3, no. 2, pp. 247-252, 2013.

[9] C. Liu, Y. Lu, and C. Wang, "GCPW-to-SIW transition using tapered slot," Proceedings of the 5thWorld Congress on Electrical Engineering and Computer Systems and Sciences (EECSS'19), Lisbon, Portugal, 2019.

[10] F. Taringou, D. Dousset, J. Bornemann, and K. Wu, "Substrate-integrated waveguide transitions to planar transmission-line technologies," in 2012 IEEE/MTT-S International Microwave Symposium Digest, 2012, pp. 13.

[11] S. Lee, S. Jung, and H. Lee, "Ultra-Wideband CPW-to-Substrate Integrated Waveguide Transition Using an Elevated-CPW Section," IEEE Microwave and Wireless Components Letters, vol. 18, no. 11, pp. 746748,2008

[12] R. Kazemi, A. E. Fathy, S. Yang, and R. A. Sadeghzadeh, "Development of an ultra wide band GCPW to SIW transition," in 2012 IEEE Radio and Wireless Symposium, 2012, pp. 171-174.

[13] S. K. Sahoo, M. Adhikary, A. Biswas, and M. J. Akhtar, "GCPW to SIW Transition for Planar Excitation of Ku-Band Substrate Integrated End-Fire Antennas," in 2019 IEEE Indian Conference on Antennas and Propagation (InCAP), 2019, pp. 1-4.

[14] R. Vincenti Gatti, R. Rossi, and M. Dionigi, "Broadband Right-Angle Rectangular Waveguide to Substrate Integrated Waveguide Transition with Distributed Impedance Matching Network," Applied Sciences, vol. 9, p. 389, 2019.

[15] R. Kazemi and A. E. Fathy, "Design of single-ridge SIW power dividers with over 75\% bandwidth," in 2014 IEEE MTT-S International Microwave Symposium (IMS2014), 2014, pp. 1-3.

[16] SMP, longwipe-SMP, mini-SMP connectors. [Online]. Available: https://www.rosenberger.com/0_documents/de/catalogs/ba_communicati on/catalog_coax/02_Chapter_SMP_Longwipe-SMP_Mini-SMP.pdf

[17] E. Diaz Caballero, A. Belenguer, H. Esteban, and V. E. Boria, "Thrureflect-line calibration for substrate integrated waveguide devices with tapered microstrip transitions," Electronics Letters, vol. 49, no. 2, pp. $132-133,2013$. 